\title{
A numerical framework for optimal control of switched input affine nonlinear systems subject to path constraint
}

\author{
Pierre Riedinger ${ }^{\mathrm{a}, *}$, Irinel-Constantin Morărescu ${ }^{\mathrm{a}}$ \\ ${ }^{a}$ Université de Lorraine, CRAN, CNRS UMR 7039, 2, avenue de la forêt de Haye, 54516 \\ Vandoeuvre-lès-Nancy Cedex, France.
}

\begin{abstract}
In this paper, we address the problem of numerical implementation of optimal control for switched input affine nonlinear systems subject to path constraint. In order to properly solve the problem, a relaxed system is introduced and the connection between the solution of this system and the solution of the initial one is established. One of the main difficulties is then related to the fact that the optimal solution can be singular. We show that, using slack variables, a set of complementarity constraints can be used to take into account the singular nature of the solution. The optimal control problem is then reformulated as a constraint optimization problem over the Hamiltonian systems and solved via a direct method. This formulation does not require a priori knowledge on the structure (regular/singular) of the solution. In addition, state path constraints are included. Numerical simulations for boost, buck-boost and Flying Capacitor converters, both in continuous and discontinuous conduction mode, illustrate the effectiveness of the proposed methodology.
\end{abstract}

Key words: Singular optimal control, switched systems, complementarity systems, numerical method

\section{Introduction}

Most of the results related to the optimal control of hybrid systems deal with subsystems sharing zero as common equilibrium. In this paper, we treat the case of switched input affine non linear systems for which generally no common equilibrium can be defined. In this context, the referred targets, named operating points, are defined as the equilibria of a relaxed system - obtained by expanding the control domain to its convex hull. As a result of the applied control strategy, the average value of the state variables computed on a sliding window coincides with the target [16].

\footnotetext{
* Corresponding author

Email addresses: pierre.riedinger@ensem.inpl-nancy.fr (Pierre Riedinger), constantin.morarescu@ensem.inpl-nancy.fr (Irinel-Constantin Morărescu)
} 
It is worth noting that the optimal solution of the relaxed system can be singular as deduced from the Pontryagin Maximum Principle (PMP). Precisely, the necessary conditions provided by PMP are inconclusive on the control value when $H_{u}=0, \forall u$. This is a well known [32, 19, 23, 4] difficulty encountered in optimal control field.

The search of analytic solutions is pervasive even for low dimensional systems [27]. In this context only few methods have been proposed to numerically solve such control problems. Indirect shooting method requires a priori knowledge on the structure (regular/singular) of the trajectory [3]. This information can be achieved using direct methods [2,31] but the determination of the control lacks of accuracy. Once the information on the structure of the optimal solution is available, an accurate solution can be obtained if singular arc conditions are imposed for the singular part of the trajectory; see for example the Goddard Rocket Problem in [30].

In order to overcome this inconvenient, we propose to reformulate the control set inclusion deduced from the necessary conditions of PMP as a set of complementarity constraints and to add them to the Hamiltonian system to form a complementarity system [12]. The multivalued part of the control (corresponding precisely to the singular cases) is then concisely expressed in these complementarity constraints. At this stage, one may view the problem as a Boundary Value Problem (BVP) and try to use a shooting method. Unfortunately, such shooting methods are not appropriate to solve this BVP because of the non uniqueness of the solution.

In this paper we propose to use a direct method [2] to minimize the cost function subject to some complementarity constraints. This approach can be classified as mixed direct-indirect method since it uses both the necessary condition of PMP and a large-scale nonlinear programming problem (NLP) arising from the discretization of the cost and of the resulting complementarity systems. The main advantage of this formulation is that no information on the structure (regular/singular) of the trajectories is a priori required since this feature is implicitly captured by the values of the state and the co-state through a switching function and the set of complementarity constraints.

To improve the accuracy of numerical results, additional constraints concerning the order of the singularity are taken into account. Finally, the method is extended to path constraints case.

The paper is organized as follows. In Section 2 one gives the system description and defines the operating points. Section 3 formulates the optimal control problem derived from PMP. The singular nature of the solution is also detailed and discussed. The complementarity formalism is used to reformulate the optimal control problem in Section 4. To increase the accuracy of the control determination we add constrains related to the order of the singularities. Section 5 contains numerical simulation illustrating the results of the proposed methodology on a variety of power converters. 


\section{System Description}

The class of constraint switched input affine nonlinear systems under consideration can be described by:

$$
\begin{aligned}
& \dot{x}(t)=f(x(t))+\sum_{i=1}^{m} u_{i}(t) g_{i}(x(t)) \\
& x(0)=x_{0} \\
& c(x(t)) \leq 0
\end{aligned}
$$

where $u(t)=\left(u_{1}(t), \ldots, u_{m}(t)\right) \in \mathbb{R}^{m}$ is the control law and $x(t) \in \mathbb{R}^{n}$ represents the state value at time $t$. The maps $f(\cdot): \mathbb{R}^{n} \mapsto \mathbb{R}^{n}, c(\cdot): \mathbb{R}^{n} \mapsto \mathbb{R}^{r}$ and $g_{i}(\cdot): \mathbb{R}^{n} \mapsto \mathbb{R}^{n}, i=1, \ldots, m$ are supposed sufficiently smooth. A vector is considered positive/negative if all its components are positive/negative. We note that (3) defines the unilateral r-dimension state-path constraints .

When $u(t) \in U=\{0,1\}^{m}$ equation (1) describes a class of Switched System (SS) largely used to express the dynamics of nonsmooth mechanical systems (see $[24,25]$ and the reference there in) or electric circuits $([9])$. On the other hand when $u(t) \in \operatorname{co}(U)=[0,1]^{m}$ where $\operatorname{co}(U)$ stands for the convex hull, equation (1) describes a Relaxed System (RS). The (SS) belongs to the class of nonsmooth systems for which the notion of solution can be properly defined and generalized in the sense of Fillipov [13]. The link between the solutions of (SS) and (RS) is established by a density theorem in infinite time [17]. Let us denote $L^{\infty}([0,+\infty), U)$ the set of all essentially bounded measurable functions mapping $[0, \infty)$ to $U$. Then $L^{\infty}([0,+\infty), U)$ equipped with the essential supremum norm (i.e. $\|f\|_{\infty}=\operatorname{ess} \sup |f|$ ) becomes a Banach space. In [17] is proven that there exists a switching law $u \in L^{\infty}([0,+\infty), U)$ such that the trajectory of (RS) is approached as close as desired by the one of (SS). For this reason, the operating points set of (SS) denoted by $X_{r e f}$, is defined as the set of equilibrium points of (RS):

$$
\begin{aligned}
X_{\text {ref }}=\left\{x_{\text {ref }} \in \mathbb{R}^{n}: f\left(x_{\text {ref }}\right)+\right. \\
\left.\quad \sum_{i=1}^{m} u_{i, r e f} g_{i}\left(x_{r e f}\right)=0, u_{i, r e f} \in[0,1]\right\} .
\end{aligned}
$$

This set defines the control targets for the state of (SS).

It is worth noting that none of the controls $u_{\text {ref }} \in \operatorname{co}(U) \backslash U$ from (5) corresponding to an equilibrium $x_{r e f}$, is admissible for (SS). The outcome is that (SS) state $x$ cannot be maintained on $x_{r e f}$ by a control taking its values in $U$ (unless the time duration between switchings tends towards 0). Consequently, if the target for (SS) is an operating point $x_{r e f}$, the asymptotic behavior of the trajectories is characterized either by a cycle near $x_{r e f}$ if a dwell time condition is applied on the switchings (i.e. a lower limit exists for the time duration between switchings) or by an infinite switching sequence with a vanishing time duration between switches as $t \rightarrow \infty$. 


\section{Optimal control problem formulation}

In the sequel, we propose a methodology to numerically solve the following optimal control problem for the system defined by equations (1)-(3):

$$
\min _{u(.)} \int_{0}^{T} L\left(x(t), x_{r e f}\right) d t
$$

where $L$ is the continuously differentiable performance function, $x_{r e f}$ stands for a chosen equilibrium point in $X_{r e f}$ and $T$ refers to the strictly positive final time and is fixed $\left(T \in \mathbb{R}^{+}\right)$.

When the (RS) has a bang-bang solution (i.e. $\left.\forall t, u(t) \in\{0,1\}^{m}\right)$, (SS) is also solved. Otherwise, it exists a non zero measure set (in the Lebesgue sense) $\mathcal{T}$ such that the optimal solution takes values $u(t) \in c o(U) \backslash U$ for all $t \in \mathcal{T}$. Although these solutions are not admissible for (SS), Pulse Wise Modulation (PWM), for example, yields an average approximation by high switching frequency [17]. Consequently we relax the optimal control problem for (SS) by searching the optimal solutions of (RS) from which suboptimal solutions for (SS) can be derived.

Suppose in a first time that no constraint are taken into account and let inspect the necessary conditions defined by the Pontryagin Maximum Principle (PMP). The Hamiltonian function $\mathcal{H}$ is given by (abnormal case is not taken into account):

$$
\mathcal{H}(t)=\lambda^{\top}(t)(f(x)+g(x) u(t))+L\left(x(t), x_{r e f}\right)
$$

where $\lambda(t) \in \mathbb{R}^{n}$ depicts the co-state whose dynamic is given by the Hamiltonian system $\dot{\lambda}(t)=-\mathcal{H}_{x}(t)\left(\dot{x}(t)=\mathcal{H}_{\lambda}(t)\right)$.

Optimality condition of PMP that $u$ minimizes the Hamiltonian function leads to the following inclusion,

$$
u_{i}(t) \in\left\{\begin{array}{l}
0 \text { if } \phi_{i}(t)>0 \\
{[01] \text { if } \phi_{i}(t)=0} \\
1 \text { if } \phi_{i}(t)<0
\end{array} \quad \forall i=1, \ldots, m\right.
$$

where $\phi(t)=\mathcal{H}_{u}(t)=\lambda^{T}(t) g(x(t))$ defines the switching function. When $u_{i}(t)=0$ or 1 , the control is called regular. A singularity arises when a component of $\phi_{i}(t)$ vanishes identically on a time interval $[a, b], b>a$. In this case, the PMP is inconclusive concerning the control value $u_{i}(\cdot)$ on $[a, b]$. This situation is referred to as singular control $[32,8,4]$ and it corresponds here to the case where $u(t)$ takes values in $c o(U) \backslash U, t \in[a, b]$. We can conclude that solution segments for (RS), which are not admissible for (SS), involve a singular control.

Nevertheless, there exist generic cases where the necessity to take into account singular arcs can be avoided when a generic property, as defined in [10], is satisfied by the control-affine system. Indeed, if the system is generic and if $m \geq 2$, it is proved that the control system (1) does not admit any singular 
trajectory. However our paper is focussing on systems that do not satisfy the generic property.

In the sequel, in order to simplify the notation we shall often avoid to emphasize the time dependency of the variables and controller.

Definition 1. For a single control $(m=1)$, if $\phi$ vanishes identically on the time interval $[a, b], b>a$ we say that $x$ belongs to a singular arc for all $t \in[a, b]$. These arcs are characterized by:

$$
\mathcal{H}_{u}(x, \lambda, u)=0, \forall u \in c o(U), \forall t \in(a, b) .
$$

To determine these arcs, successive time differentiations:

$$
\frac{d^{q}}{d t^{q}} \mathcal{H}_{u}(x, \lambda, u)=0
$$

are required. The differentiation has to be done until at least a component of the control appears. The smallest positive integer $q^{*}$ which is always even, such that

$$
\frac{\partial}{\partial u}\left(\frac{d^{q^{*}}}{d t^{q^{*}}} \mathcal{H}_{u}(x, \lambda, u)\right) \neq 0,
$$

if it exists, is called the order of singularity [23] (or index of the DAE). As we have shown in [27], for low dimensional systems (i.e. $n \leq 3$ ), the analytic expression of $u$ can be explicitly determined by using the set of algebraic equations (10) and a second order necessary condition given by the Generalized Legendre-Clebsh Condition $[32,23,19]$ :

$$
(-1)^{\frac{q^{*}}{2}} \frac{\partial}{\partial u}\left(\frac{d^{q^{*}}}{d t^{q^{*}}} \mathcal{H}_{u}(x, \lambda, u)\right) \geq 0 .
$$

On one hand, to determine analytically the optimal solutions including singular arcs is a particularly hard problem and only low dimensional systems (i.e. $\mathrm{n}=2,3$ ) have been treated [27]. Moreover, knowledge on the singular arcs are of few help in determination of the junction time between singular and regular arcs. On the other hand, standard indirect numerical methods such as multiple shooting methods are not appropriate to deal with singular arcs without a priori information on the structure of the trajectories (see [14], [22] and [28] to apply multiple shooting methods in this context). This information can be achieved using regularization techniques such as the continuation method used in [26], [18], [21]. In this paper, we propose to use direct method and an ad hoc formulation that takes implicitly into account the singular arcs and do not require a priori information on the solution structure.

\section{Optimal control using complementarity system formalism}

Complementarity is a useful concept that can be used to describe properly the behavior of nonsmooth systems. Complementarity systems have been largely employed in the analysis and control of mechanical systems $[5,7,25]$ and recently they are used to describe the dynamics of electric circuits $[9,34,1]$. 
Definition 2. A Linear Complementarity Problem (LCP) is a system given by:

$$
\left\{\begin{array}{l}
y \geq 0 \\
A y+b \geq 0 \\
y^{T}(A y+b)=0
\end{array}\right.
$$

which is compactly rewritten as

$$
0 \leq y \perp A y+b \geq 0
$$

Such an LCP has a unique solution for all $b$ if and only if $A$ is a P-matrix, i.e. all its principal minors are positive [12].

Combining complementarity conditions with differential equations we get what usually in the literature is referred to as complementarity systems (see [6] and the references therein):

$$
\left\{\begin{array}{l}
\dot{x}(t)=f(x(t), u(t)) \\
y(t)=h(x(t), u(t)) \\
0 \leq y(t) \perp u(t) \geq 0
\end{array}\right.
$$

We also note that in some applications, the complementarity system may contain an external input (forcing term) or the adjoint variable [33]. In the sequel, we propose to reformulate the Hamiltonian system and the necessary condition (8) as a complementarity system.

\subsection{Relay inclusion as a complementarity constraint}

Let us recall (see [33]) that the relay-type function (8) can be rewritten as a complementarity constraint and an algebraic equation as follows:

$$
\begin{aligned}
& 0 \leq s_{1} \perp u \geq 0 \\
& 0 \leq s_{2} \perp(1-u) \geq 0 \\
& 0=s_{1}-s_{2}-\phi
\end{aligned}
$$

where $s_{1}$ and $s_{2}$ are m-dimensional vectors and define the positive and the negative part of $\phi(x, \lambda)$. In other words, the components of the two vectors $s_{1}$ and $s_{2}$ are defined by:

$$
\begin{aligned}
& s_{1 i}(t)=\left\{\begin{array}{l}
0 \text { if } \phi_{i}(t)<0 \\
\phi_{i}(t) \text { if } \phi_{i}(t) \geq 0
\end{array} \quad \forall i=1, \ldots, m\right. \\
& s_{2 i}(t)=\left\{\begin{array}{l}
-\phi_{i}(t) \text { if } \phi_{i}(t)<0 \\
0 \text { if } \phi_{i}(t) \geq 0
\end{array}\right.
\end{aligned}
$$

It is important to note that as long as $s_{1 i}>0$ or $s_{2 i}>0$ the component $u_{i}$ of the control law $u$ is uniquely determined. When $s_{1 i}=0 \& s_{2 i}=0$ the uniqueness of $u_{i}$ is lost and the choice of its value is a matter of optimization. We also observe that the situation $s_{1 i} \cdot s_{2 i}>0$ cannot occur since this will contradict either (16) or (17). 


\subsection{Path constraints}

Until now, no consideration has been made on the path constraints (3). Like for singular controls, the index $\hat{q}$ of the state constraint is the first time where $u$ appears explicitly in the successive time differentiation of $c$ along the trajectories. This index allows to define the mixed control-state path constraints occurring on the boundary $c(x)=0$ :

$$
p(x, u)=\frac{d^{\hat{q}}}{d t^{\hat{q}}} c(x) \leq 0 .
$$

and the Lagrangian function:

$$
\mathcal{L}(x, u, \lambda, \mu)=\mathcal{H}(x, u, \lambda)+\mu^{T} p(x, u)
$$

where $\mu \geq 0$ represents the $r$-dimensional vector of Lagrange multipliers.

Assumption 1. We assume in the sequel, that $\hat{q}=1$.

When it is not the case, the co-state might be only a measure and the given version of PMP does not apply [11].

Theorem 1. If $u^{*}$ is the optimal control for the state constraint system (13) when the cost is defined by (6) then $u^{*}$ satisfies the following constraint optimization problem:

$$
\begin{aligned}
\mathcal{C O P}: & \min _{u(\cdot)} \int_{0}^{T} L\left(x(t), x_{r e f}\right) d t \\
\text { s.t. } \dot{x} & =\mathcal{L}_{\lambda} \quad \dot{\lambda}=-\mathcal{L}_{x} \\
x(0) & =x_{0} \quad \lambda(T)=0 \\
0 & \leq s_{1} \perp u \geq 0 \\
0 & \leq s_{2} \perp(1-u) \geq 0 \\
0 & =s_{1}-s_{2}-\mathcal{L}_{u}(x, \lambda, \mu) \\
0 & \leq \mu_{i} \perp\left(s_{1}+s_{2}\right)_{j} \geq 0, i=1, \cdots, r j=1, \cdots, m \\
0 & \leq \mu \perp-c(x) \geq 0
\end{aligned}
$$

Proof. These necessary conditions are directly deduced from PMP in case of state constraint (Chap.6; [29]) and in presence of complementary constraints as explained in the following comments:

- Generally when path constraints are considered, jumps occur on the costate at junction time $\tau$. They are characterized by $\lambda^{+}(\tau)=\lambda^{-}(\tau)+$ $\alpha \nabla c(x(\tau))$. In fact, $\alpha$ can be taken equal to zero when the motion follows the border $c(x)=0$ for $t \geq \tau$ as proven in the pioneer work (Chap.6; [29]).

- When $\mu$ equals zero, (20) allows us to retrieve the unconstraint version of PMP. Note that when $\mu=0,(26)$ is equivalent to (18). The complementarity relation (28) assures that $\mu=0$ when $c(x)$ is not active (i.e. $c(x)>0)$. 
- The constraint $c_{i}(x)$ is activated (i.e. $c_{i}(x)=0$ ) when $\mu_{i}$ becomes positive and modifies the co-state dynamics as it is required (Chap.6; [29]).

- The complementarity conditions $0 \leq \mu_{i} \perp\left(s_{1}+s_{2}\right)_{j} \geq 0$ are necessary for any $i=1, \cdots, r$ and for any $j=1, \cdots, m$ to ensure the necessary condition $\mathcal{L}_{u}=0$ when some components of the path constraints are active. Indeed, if at least one of the constraints is strongly active, says the $i t h$, then $\mu_{i}>0$ and $s_{1}=s_{2}=0$ and all components of $u \in\left[\begin{array}{ll}0 & 1\end{array}\right]^{m}$ are subject to $\mathcal{L}_{u}=0$ as required by the minimization of the Lagrangian $\mathcal{L}$ (augmented Hamiltonian).

It has to be mentioned here two important features of the above formalism. Firstly, this formulation clearly takes into account both singular arcs and path constraints. Secondly, no assumption is made on the structure (singular/regular) of the trajectories. This is implicitly captured by the values of $x$ and $\lambda$ through the switching function $\phi$ and the complementarity constraints. Consequently, we do not require any initial information on the existence of singular arcs entering in the optimal trajectory.

The optimization problem $(\mathcal{C O P})$ can be solved using direct method i.e. by minimizing the cost function (21) subject to the complementarity system (22-28). Of course, in order to determine accurate solution, recall that it is important to scale the problem and to use code that use multiple phase formulation to reduce the sensitivity and that enable grid refinement procedure. Among the possible alternatives, for all the examples given in the next section, we have used the beta version GPOPS Toolbox for Matlab. This toolbox solves multiphase optimal control problems [2] and is based on Radau pseudo spectral methods to parametrize the solution. In this orthogonal collocation method, the collocation points are the Legendre-Gauss-Radau points [15] and an interesting feature is that it is a Gaussian quadrature implicit integration scheme. From a practical point of view, complementary constraints is achieved by penalization terms in the cost of the form $\rho y w$ if $0 \leq y \perp w \geq 0$, with weight $\rho>0$ and chosen sufficiently large.

Remark 1. The proposed numerical framework does not catch Fuller or zeno phenomena [20]. These phenomena characterized by an infinite number of switchings in finite time which can occur when the trajectories hits the path constraint or the singular surfaces, are not taken into account by the proposed numerical method. In fact, these phenomena is avoided by the discretization of the problem and is ultimately approximated.

A practical and significant improvement in minimizing the cost function (21) subject to the complementarity system (22-28) can be obtained by including the algebraic constraints (10) for $q=1, \cdots, q^{*}$. The last constraint when $q=q^{*}$ is particularly beneficial since the control appears in it explicitly. Of course, as these constraints correspond to the case where the control is singular, these additions are only possible when path constraints are not active (that is when 
$\mu=0$ and $\dot{\lambda}=-\mathcal{H}_{x}=-\mathcal{L}_{x}$ ). If the path constraints are active, they are unnecessary since $u$ appears this time in the path constraints.

Theorem 2. If $u^{*}$ is the optimal control of problem defined by cost (6) and state constraint system (1-3) then $u^{*}$ satisfies $\mathcal{C O P}$ of Theorem 1 and the following additional constraints related to the order of the singularity (10):

$$
\begin{aligned}
& 0=s_{2 q+1}-s_{2 q+2}-\frac{d^{q}}{d t^{q}} \phi, q=1, \cdots, q^{*} \\
& 0 \leq \prod_{i=1}^{r} c_{i}(x) u(1-u) \perp\left(s_{2 q+1}+s_{2 q+2}\right) \geq 0 \\
& 0 \leq s_{2 q+1} \perp s_{2 q+2} \geq 0
\end{aligned}
$$

where $s_{2 q+1}, s_{2 q+2} q=1, \cdots, q^{*}$ are additional $m$-dimension slack variables.

Proof. It is easy to show from equation (29) and (31) that $s_{2 q+1}$ and $s_{2 q+2}$ are respectively the positive and the negative part of $\frac{d^{q}}{d t^{q}} \phi$. Then, (30) imposes that the $i^{t h}$ component of $\left|\frac{d^{q}}{d t^{q}} \phi\right|$ equals zero when $u_{i}$ is singular and the state $x$ does not satisfy one of the constraints given by (3). Following singular arc definition, these relations are fulfilled and their inclusion in $(\mathcal{C O P})$ improves the numerical solution notably in the control determination as we will show in the next section.

\section{Application to power converters}

DC-DC converters such as buck, boost, buck-boost, flying capacitor converters enter clearly in the class of constraint switched affine systems. We have applied our numerical approach for all these converters and we present in the sequel two selected cases to illustrate the effectiveness of the proposed procedure.

\subsection{FCC converter}

In the case of a few megawatt industrial power applications, the classical power converters have a very high voltage in the switching components (several kilovolts). To compensate this, a new class of power converters called Flying Capacitor Converters (FCC) appeared (or multicellular converter). The structure of the FCC converter reduces the voltage throughout the switches. It is composed of serial connections between semiconductor switching devices and passive storage elements to achieve the target operating voltage as illustrated in Figure 1.

Three switching cells can be isolated, each of them being able to operate in two complementary mode. The behavior of each cell can be described using only one boolean control variable $u_{i} \in\{0,1\}$ with $i=1,2,3$. $u_{i}=1$ means that the upper switch is closed and the lower switch is open whereas $u_{i}=0$ means that the upper switch is open and the lower switch is closed (Figure 1). 


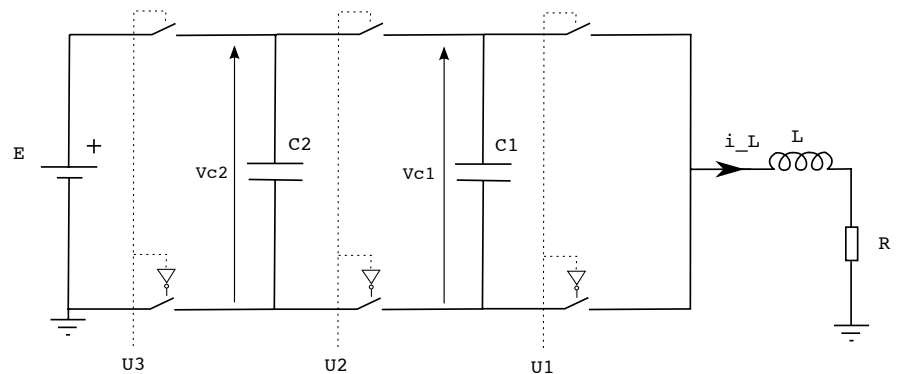

Figure 1: Fying capacitor converter

The state equations of the converter have an affine form given by

$$
\left[\begin{array}{c}
\dot{x}_{1} \\
\dot{x}_{2} \\
\dot{x}_{3}
\end{array}\right]=\left[\begin{array}{c}
0 \\
0 \\
-\frac{R}{L} x_{3}
\end{array}\right]+\left[\begin{array}{ccc}
-\frac{x_{3}}{C_{1}} & \frac{x_{3}}{C_{1}} & 0 \\
0 & -\frac{x_{3}}{C_{2}} & \frac{x_{3}}{C_{2}} \\
\frac{x_{1}}{L} & \frac{x_{2}-x_{1}}{L} & \frac{E-x_{2}}{L}
\end{array}\right]\left[\begin{array}{l}
u_{1} \\
u_{2} \\
u_{3}
\end{array}\right]
$$

where $x_{1}, x_{2}$ are the voltage on each capacitor and $x_{3}$ the load current. The chosen parameters are $E=30 \mathrm{~V}, R=10 \Omega, C_{1}=C_{2}=40 \mu \mathrm{F}$ and $L=10 \mathrm{mF}$.

The capacitor voltages must be balanced to $2 E / 3$ and $E / 3$, while the demanding load current is fixed to $2 \mathrm{~A}$. Then, $x_{\text {ref }}=\left[\begin{array}{lll}E / 3 & 2 E / 3 & 2\end{array}\right]^{T}$.

The optimization problem consists in minimizing the quadratic criteria:

$$
\min _{u(\cdot)} \int_{0}^{T}\left(x-x_{r e f}\right)^{\prime} Q\left(x-x_{r e f}\right) d t
$$

The weight matrix $Q$ is taken to: $Q=\left[\begin{array}{ccc}0.2 & -0.1 & 0 \\ -0.1 & 0.1 & 0 \\ 0 & 0 & 200\end{array}\right]$

Figure 2 and 3 show the control and state trajectories starting from the origin. The objective value is $J=0.129$. As expected, the control are totally singular and are properly determined using the proposed formulation. In comparison, a direct transcription of equation (6) and (1-2) using the same GPOPS toolbox, yields the results provided in Figure 4 and Figure 5 . The solver returns a flag indicating that the optimization has finished successfully and that the optimality conditions are satisfied. In fact, we can observe that the trajectories are similar but the control is far from being accurate due to Hamiltonian singularities. All these simulations have been done with the same parameters: 3 phases formulations, 3 mesh iterations, analytic derivative provided and the same mesh tolerance (1e-3), see [2], [15] and website dedicated to GPOPS for more explanation. For this example, there is no path constraint. So, the differences in the numerical results are determined by the adopted formulation. In view of the results, the Karush-Kuhn-Tucker conditions used to solve directly the problem (6) and (1-2) does not allow to determine properly the control in singular cases while the additional algebraic constraints given by Theorem 1 and 2 force this determination. 

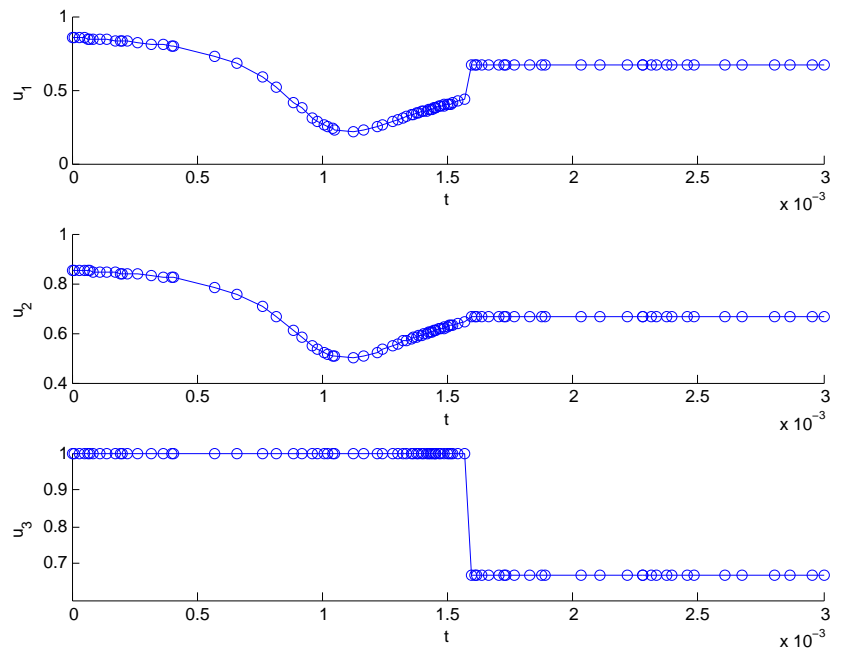

Figure 2: Optimal singular controls using Hamiltonian and complementarity constraints
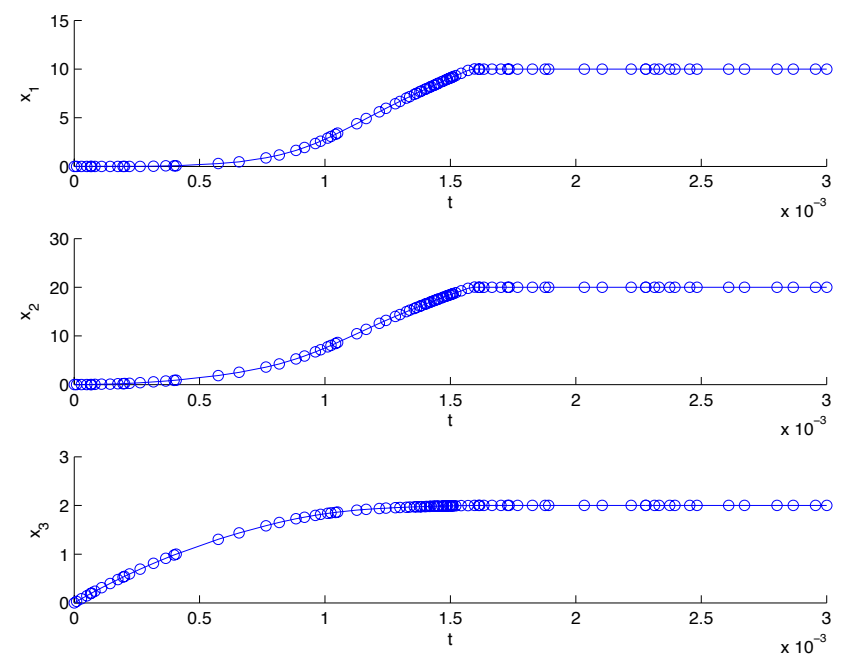

Figure 3: Optimal state trajectories using Hamiltonian and complementarity constraints 

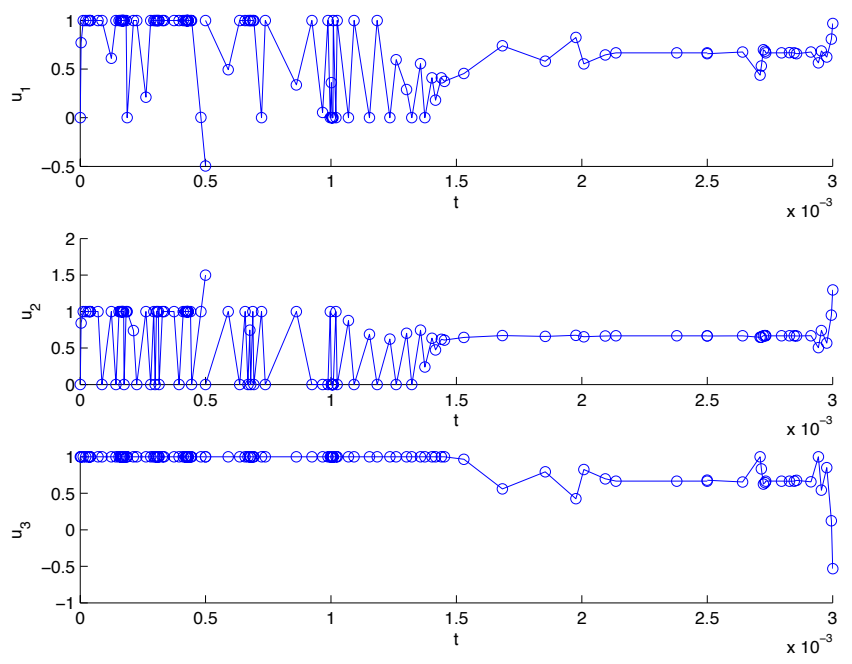

Figure 4: Numerical results in a naive transcription : optimal controls
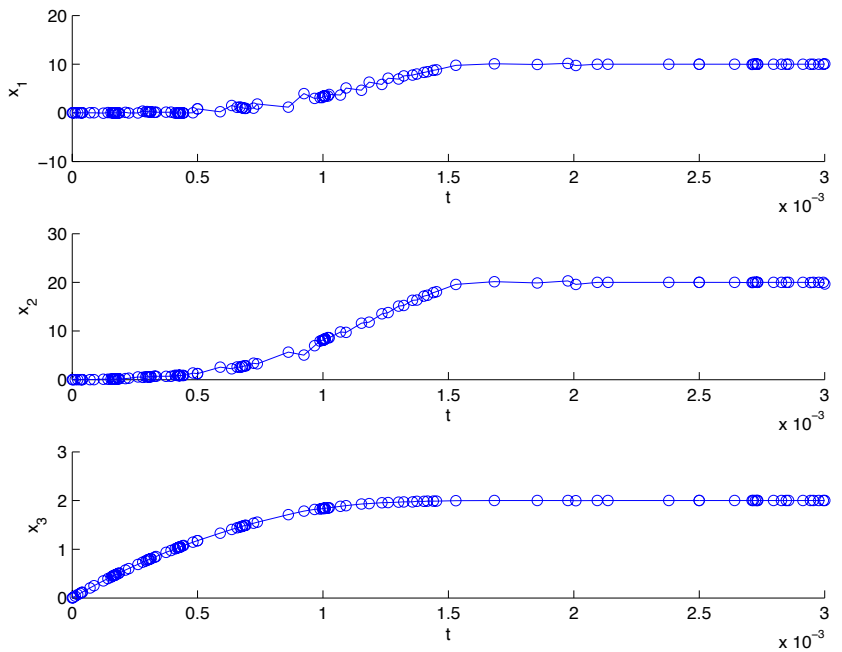

Figure 5: Numerical results in a naive transcription : state trajectories 


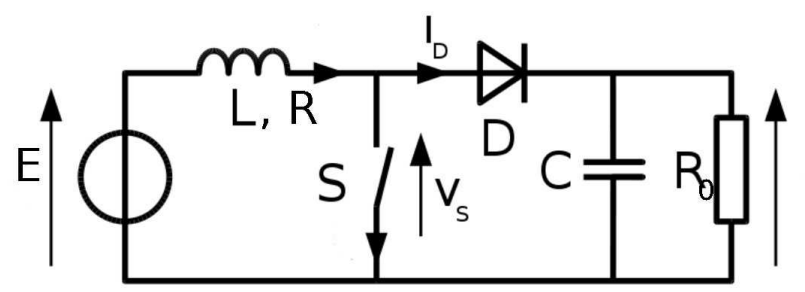

Figure 6: Boost converter

\subsection{Boost converter}

The topology of a boost converter is given in Figure (6). In continuous conduction mode, following the position of the switch $u$, two subsystems $\dot{x}=$ $A_{i} x+B_{i}, i=1,2$ can be considered with matrices $A_{1}=\left(\begin{array}{cc}\frac{-R}{L} & 0 \\ 0 & \frac{-1}{R_{0} C}\end{array}\right)$, $A_{2}=\left(\begin{array}{cc}\frac{-R}{L} & \frac{-1}{L} \\ \frac{1}{C} & \frac{-1}{R_{0} C}\end{array}\right), B_{1}=\left(\begin{array}{c}\frac{E}{L} \\ 0\end{array}\right)$ and $B_{2}=B_{1}$. Generally the control strategies proposed in the literature do not take into account the discontinuous mode that may appears when the demanding current in the load is low. In this case, the current in the diode vanishes and produces an autonomous switch. Few results concerning optimal control of hybrid systems are related to the case of autonomous switches. The third subsystem is then described by

$$
A_{3}=\left(\begin{array}{cc}
0 & 0 \\
0 & \frac{-1}{R_{0} C}
\end{array}\right), \text { and } B_{3}=\left(\begin{array}{l}
0 \\
0
\end{array}\right)
$$

In fact, it is not necessary to add this last subsystem when $x_{1}=0$ since there exists an equivalent control $u_{e q}(x) \in\left[\begin{array}{ll}0 & 1\end{array}\right]$ which forces $\dot{x}_{1}=0$ and such that the dynamics of $\dot{x}=u_{e q}\left(A_{1} x+B_{1}\right)+\left(1-u_{e q}\right)\left(A_{2} x+B_{2}\right)$ matches the one of the third subsystem. So, the optimal control problem is solved with path constraint corresponding to $x_{1} \geq 0$.

The parameters are chosen as follow: $E=20 \mathrm{~V}, R_{0}=50 \Omega, R=1 \Omega$, $L=2 m H$ and $C=100 \mu F$.

The optimization problem consists in minimizing, for a given equilibrium $x_{r e f}$, the quadratic criteria:

$$
\min _{u(.)} \int_{0}^{T}\left(x-x_{r e f}\right)^{\prime} Q\left(x-x_{r e f}\right) d t
$$

We have applied the following scenario: Starting from zero initial condition, the state is steered to equilibrium $x_{r e f}=(2.2,44.4)$ obtained for $u_{r e f}=0.6$ then, after $5 \mathrm{~ms}$ a new reference $x_{r e f}=(0.78,27.46)$ corresponding to $u_{r e f}=0.3$ is applied.

Figures 8 and 7 show the state trajectories and the optimal control as well as the Lagrange multiplier obtained for the weight matrix $Q=\operatorname{diag}(1,10)$. The 

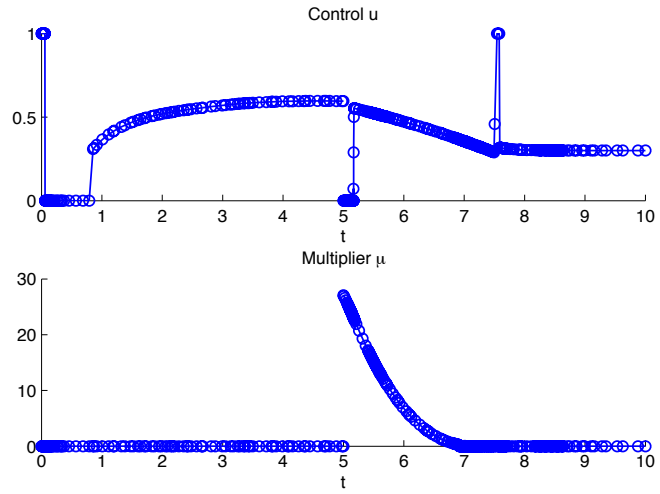

Figure 7: Boost converter: Optimal controls
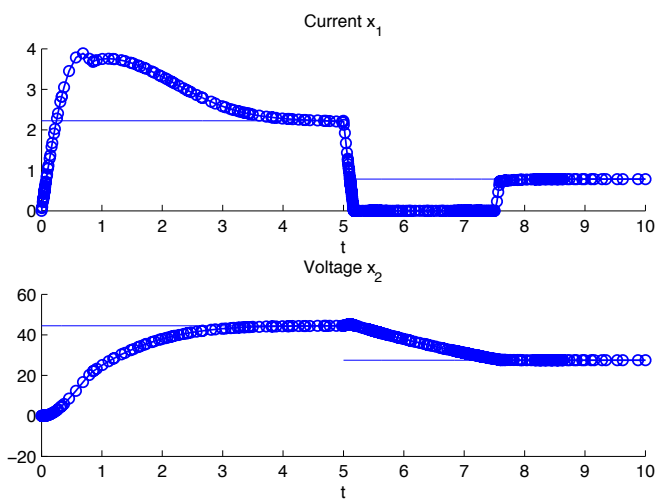

Figure 8: Boost converter : State trajectories 
time scale is expressed in $m s$. The numerical results show that just at the beginning of the simulation the control switches from 1 to 0 before the optimal trajectory reaches the singular arc which happens after approximatively $1 \mathrm{~ms}$. When a new reference is applied at time $t=5 \mathrm{~ms}$, control switches to zero and the system enters in discontinuous conduction mode when the current vanishes. The value of the Lagrangien multiplier clearly indicates this while the control values allow to meet the constraint $\dot{x}_{1}=0$. The system returns in continuous conduction mode with a short activation of mode 2 at time $t=7.5 \mathrm{~ms}$. The control becomes singular once the reference is reached.

Now, Figure 9 compares the precision with which the optimal control is determined according to the formulation adopted to solve the optimal control problem. Starting from the origin, the cost function consists to minimize the quadratic criteria $\min _{u(.)} \int_{0}^{T}\left(x-x_{r e f}\right)^{\prime} Q\left(x-x_{r e f}\right) d t$ where $x_{r e f}$ corresponds to the equilibrium given by $u_{r e f}=0.5$.

At the bottom (c), a direct transcription into a NLP leads to poor results as expected when the control is singular. A gap in the accuracy is obtained with a complementarity formulation like in Theorem 1 as it is shown in the (b) part of the Figure 9. A full improvement is reached in the top of Figure 9 (a) when additional constraints associated to the order of the singularity (10) are taken into account (Theorem 2). Thus, this example reflects the ability of the formulation given in Theorem 2 to determine properly the optimal control without any information on the structure of the solution.

\section{3. buckboost converter}

The last example concerns a buck-boost converter and it is chosen to show that this numerical approach can be useful not only to compute optimal openloop control (in case of predictive control for example) but also to design robust close-loop controller.

In Figure 10 the red circle is the reference and the optimal trajectories starting from different initial conditions are plotted in blue. We can observe that discontinuous conduction mode appears along the negative part of $x_{2}$-axis and that all the trajectories reach the target $x_{r e f}$ following a singular arcs. As Figure 10 shows, the state space is partitioned following the control to be used. It can be observed that the singular trajectories, at the junction between the different parts, defined optimal sliding surfaces.

Clearly, there is a partition that defines $u$ as a state feedback $u(x)$. We think that this partition can be achieved using learning machines such as neural networks.

The parameters used in the simulations above are: $E=R=L=C=1$, $A_{1}=\left(\begin{array}{cc}0 & 0 \\ 0 & \frac{-1}{R C}\end{array}\right) A_{2}=\left(\begin{array}{cc}0 & \frac{1}{L} \\ \frac{-1}{C} & \frac{-1}{R C}\end{array}\right) B_{1}=\left(\begin{array}{c}\frac{E}{L} \\ 0\end{array}\right) B_{2}=\left(\begin{array}{l}0 \\ 0\end{array}\right)$ weight matrice $Q=\operatorname{diag}\left(q_{1}, q_{2}\right)$ with $q_{1}=1$ and $q_{2}=10$.

\subsection{How to implement suboptimal switched control law?}

The optimal controls determined in this paper take their values in $c o(U)$ and are generally (when singular) not admissible for the original switched systems 

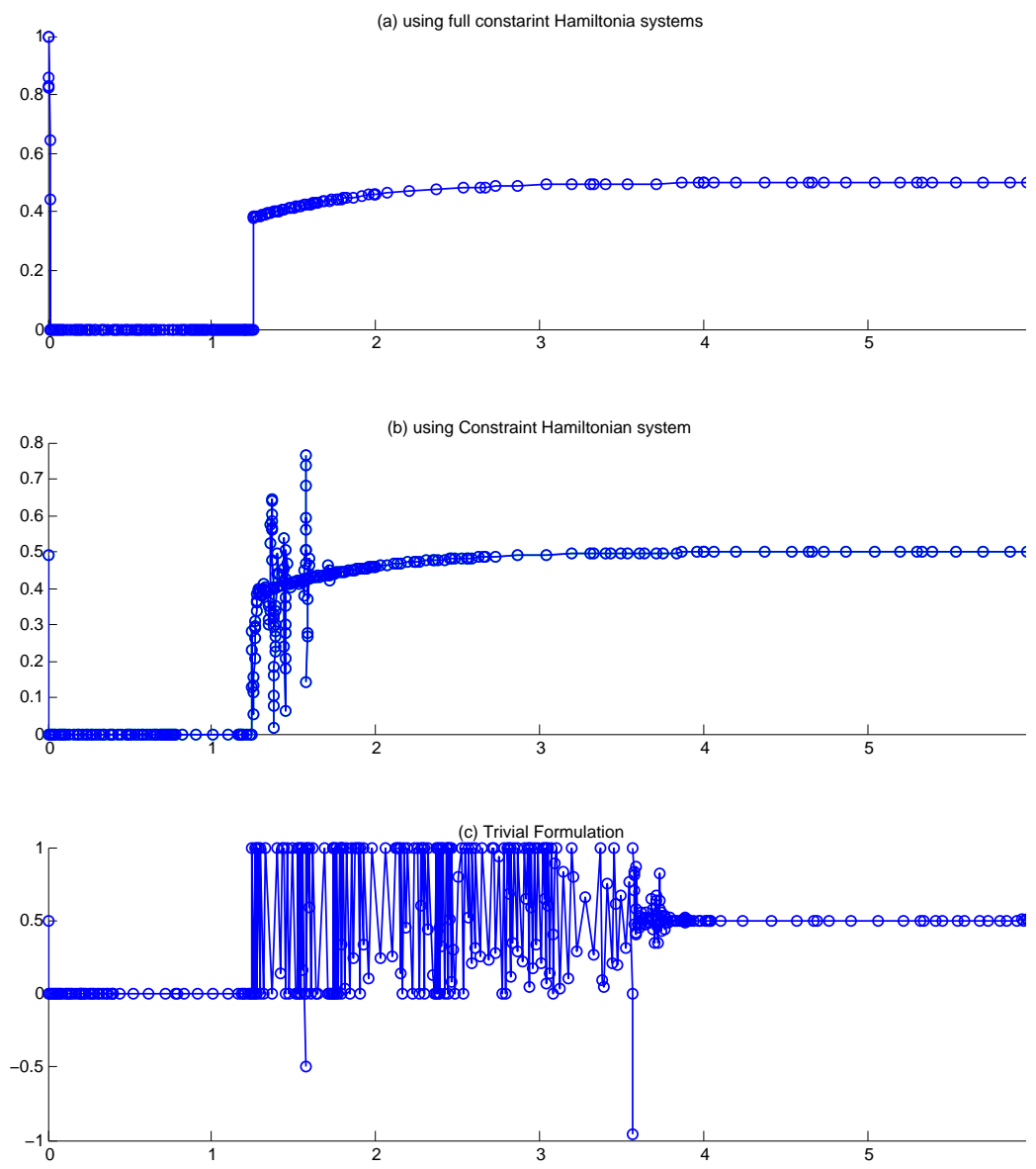

Figure 9: Boost converter: Improvement in the numerical control determination; c: direct transcription, b: Hamiltonian like a Complementarity Problem; a: Full Constraint formulation using order of the singularity 


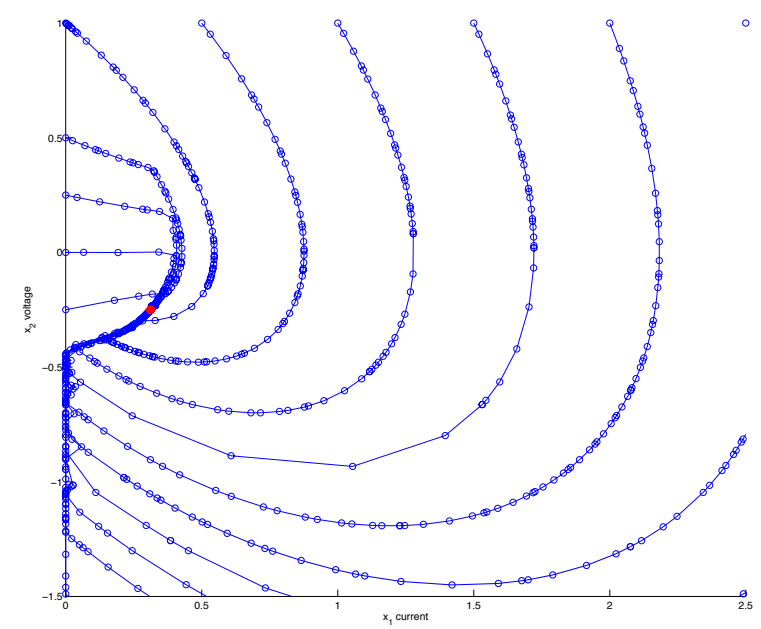

Figure 10: BuckBoost converter: Optimal trajectories in state space. $x_{r e f}$ : red circle

(SS). The density theorem given in [17] shows that all trajectories of the relaxed system (RS) can be approached as close as desired by the one of the switched systems (SS). So, the optimal one, too. In order to approach an optimal trajectory of (RS) by the one of (SS) can be simply obtained using a Pulsewise Modulation technic (PWM) to generate the switching law as it is well known in the field of power electronic. In the ideal case, higher switching frequency $f_{s}$ leads to better approximations of the optimal trajectory. This fact explains also why (SS) has only suboptimal solutions when the optimal solution of (RS) possesses singular arcs. The limit of the switching law sequence $u\left(t, f_{s}\right) \in U$ obtained by increasing $f_{s} \rightarrow+\infty$, is the singular control almost everywhere.

\section{Conclusion}

In this paper we have proposed a numerical framework to solve optimal control problem for switched input affine nonlinear systems. One specific feature of this class is that singular controls have to be considered in order to obtain the optimal solution. As singular controls are determined by an inclusion depending on the state and the co-state, we have reformulated the optimization problem using the augmented (in case of path constraints) Hamiltonian system and a set of slack variables defining a set of complementarity constraints. This constraint augmented optimization problem is then solved with a direct method dedicated to optimal control.

The contribution of the proposed methodology is twofold: an accurate optimal control is determined without any additional information concerning the 
existence and the number of the singular arcs belonging to the optimal trajectory and, the optimal control is determined even in the presence of autonomous switches. The first contribution is an important relaxation since generally in the provided singular control examples in the literature, an a priori knowledge of the number of singular arcs is required (see for example the Godart Rocket example). The second contribution allows to complete the simulation of electric circuits containing switching elements, including the discontinuous conduction mode.

It is noteworthy that a direct transcription of the optimal control problem as a non linear program leads to poor results as the FCC converter example has shown. The effectiveness of our method has been illustrated in several examples.

\section{Acknowledgments}

This work was partially supported by ANR project ArHyCo, Programme "Systèmes Embarqués et Grandes Infrastructures"- ARPEGE, contract number ANR-2008 SEGI 004 01-30011459. The research leading to these results has received funding from the European Community's Seventh Framework Programme (FP7/2007-2013) under grant agreement $\mathrm{n}^{\circ}$ 257462: HYCON2 Network of Excellence "Highly-Complex and Networked Control Systems", and from Contrat de Projet État-Région 2007-2013, Région Lorraine.

\section{References}

[1] V. Acary, O. Bonnefon, B. Brogliato, Time-stepping numerical simulation of switched circuits with the nonsmooth dynamical systems approach, IEEE Transactions on Computer-Aided Design of Integrated Circuits and Systems 29 (7) (2010) 1042-1055.

[2] J. T. Betts, Practical methods for optimal control using nonlinear programming, Advanced design and control, Siam, 2001.

[3] F. Bonnans, P. Martinon, E. Trelat, Singular arcs in the generalized goddard's problem, Journal of optimization theory and applications 139 (2) (2008) 439-461.

[4] B. Bonnard, C. Bernard, M. Chyba, Singular Trajectories and their Role in Control Theory, Series: Mathématiques et Applications, Springer SMAI, 2003.

[5] B. Brogliato, Nonsmooth Mechanics, Springer CCES, London, 1999.

[6] B. Brogliato, Some perspectives on the analysis and control of complementarity systems, IEEE Transactions on Automatic Control 48 (6) (2003) 918-935. 
[7] B. Brogliato, R. Lozano, B. Maschke, O. Egeland, Dissipative Systems Analysis and Control. Theory and Applications, 2nd ed., Springer CCES, London, 2007.

[8] A. Bryson, Y.-C. Ho, Applied optimal control, Taylor \& Francis, 1975.

[9] M. Çamlibe, W. Heemels, J. Schumacher, Stability and controllability of planar bimodal linear complementarity systems, in: 42nd, IEEE Conference on Decision and Control, Maui, Hawaii USA, 2003.

[10] Y. Chitour, E. Trélat, Singular trajectories of control-affine systems, SIAM J. Control Optim. 47 (2) (2008) 1078-1095.

[11] F. Clarke, Optimization and Nonsmooth Analysis, SIAM, 1990.

[12] F. Facchinei, J. Pang, Finite-dimensional Variational Inequalities and Complementarity ProblemsResearch, New-York, (2003), Springer Series in Operations Research, New-York, 2003.

[13] A. Filippov, Differential equations with discontinuous righthand sides, vol. 18, Mathematics and its Applications (Soviet Series), 1988.

[14] G. Fraser-Andrew, Finding candidate singular optimal controls: A state of the art survey, Journal of Optimization Theory and Applications 60 (2) (1989) $173-190$.

[15] D. Garg, M. A. Patterson, W. W. Hager, A. V. Rao, D. A. Benson, G. T. Huntington, A unified framework for the numerical solution of optimal control problems using pseudospectral methods, Automatica 46 (11) (2010) 1843-1851.

[16] P. Hauroigné, P. Riedinger, C. Iung, Switched affine systems using sampleddata controllers: robust and guaranteed stabilization, IEEE Transactions on Automatic Control.

[17] B. Ingalls, E. D. Sontag, Y. Wang, An infinite-time relaxation theorem for differential inclusions, Proceedings of the 2002 American Mathematical Society 131 (2) (2003) 487-499.

[18] D. Jacobson, S. Gershwin, M. Lele, Computation of optimal singular controls, IEEE Transaction on Automatic Control 15 (1) (1970) 67 - 73.

[19] A. Krener, The higher order maximal principle and its application to singular extremals, SIAM Journal of Control and Optimization 15 (2) (1977) 256-293.

[20] I. Kupka, The ubiquity of Fuller's phenomenon Nonlinear controllability and optimal control, Textbooks Pure Appl. Math., Dekker, 1990. 
[21] P. Martinon, Numerical resolution of optimal control problems by a pieciwise linear continuation method, Ph.D. thesis, Institut National Polytechnique de Toulouse (2005).

[22] H. Maurer, Numerical solution of singular control problems using multiple shooting techniques, Journal of Optimization Theory and Applications 18 (2) (1976) 235-257.

[23] V. Michel, Singular optimal control - the state of the art, Tech. rep., University of Kaiserslautern (1996).

[24] J. J. Moreau, Unilateral contact and dry friction in finite freedom dynamics, vol. 302, Springer-Verlag, 1998.

[25] I.-C. Morărescu, B. Brogliato, Passivity-based tracking control of multiconstraint complementarity lagrangian systems, IEEE Transactions on Automatic Control 55 (6) (2010) 1300-1310.

[26] P. J. Moylan, J. B. Moore, Generalizations of singular optimal control theory, Automatica 7 (5) (1971) pp. 591-598.

[27] D. Patino, P. Riedinger, C. Iung, Practical optimal state feedback control law for continuous-time switched affine systems with cyclic steady state, Int. J. Control 82 (7) (2009) $1357-1376$.

[28] P.Martinon, F.Bonnans, E.Trélat, Singular arcs in the generalized goddard's problem, Journal of Optimization Theory and Applications 139 (2) (2008) 439-461.

[29] L. Pontryagin, V. Boltyanskii, R. Gamkrelidze, E. Mishchenko, Théorie mathématique des processus optimaux, Editions MIR Moscou, 1974.

[30] A. V. Rao, D. A. Benson, C. L. Darby, M. A. Patterson, C. Francolin, G. T. Huntington, Algorithm 902: Gpops, a matlab software for solving multiple-phase optimal control problems using the gauss pseudospectral method, ACM Transactions on Mathematical Software 37 (2) (2010) 1-39.

[31] P. Riedinger, J. Daafouz, C. Iung, About solving hybrid control problems, in: 17th IMACS World Congress, Paris, 2005.

[32] H. Robbins, A generalized legendre-clebsch-condition for the singular cases of optimal control, IBM Journal of Research and Development 11 (4) (1967) 361-372.

[33] J. Schumacher, Complementarity systems in optimisation, Mathematical Programming, Serie B 101 (2004) 263-295.

[34] F. Vasca, L. Iannelli, M. Camlibel, R. Frasca, A new perspective for modeling power electronics converters: complementarity framework, IEEE Trans. on Power Electronics 24 (2) (2009) 456-468. 